\title{
WARFARIN-INDUCED ALOPECIA: A RARE CASE REPORT
}

\author{
SAURAB A, BALAJI O, AMITA P, NAVIN PATIL*
}

Department of Pharmacology, Kasturba Medical College, Manipal, Karnataka, India. Email: navin903@gmail.com

Received: 24 March 2017, Revised and Accepted: 07 July 2017

\begin{abstract}
As new drugs are developed everywhere worldwide to combat many diseases, side effects due to drugs are always a matter of concern. Warfarin is indicated for the treatment of venous thromboembolism over many decades and it is always associated with many side effects ranging from bleeding complications, gastrointestinal disturbances to skin necrosis. Hair fall with oral anticoagulants had always been a matter of debate and concern and so far, only very few case reports have been published on warfarin-induced hair loss. Hence, we report a case of warfarin-induced alopecia in a 39-yearold male in a tertiary care hospital in Southern India.
\end{abstract}

Keywords: Anticoagulation, Adverse drug reaction, Telogen effluvium, Hair loss.

(C) 2017 The Authors. Published by Innovare Academic Sciences Pvt Ltd. This is an open access article under the CC BY license (http://creativecommons. org/licenses/by/4. 0/) DOI: http://dx.doi.org/10.22159/ajpcr.2017.v10i10.18688

\section{INTRODUCTION}

Hair loss also known as alopecia is a very common medical condition affecting both sexes due to a wide range of etiological factors. Androgenetic alopecia (female/male), alopecia areata, telogen effluvium (TE), anagen effluvium, trichotillomania, alopecia related conditions such as seborrheic dermatitis, lichen planus and discoid lupus erythematosus, tinea capitis, impetigo, secondary syphilis, thyroid disease, and iron deficiency are some of wellknown conditions associated with hair loss [1]. Many drugs are also implicated in causing hair loss which includes anticoagulants, interferon alfa, antidepressants, antitubercular drugs, tyrosine kinase inhibitors, and anticancer drugs [2]. Hence, we report a case of warfarin-induced alopecia in a 39-year-old patient who underwent mechanical heart valve replacement in a tertiary care hospital in Southern India.

\section{CASE REPORT}

Informed consent was taken from the patient. A 39-year-old female patient, known case of mechanical heart valve replacement on warfarin therapy since 2008 came with complaints of hair fall. On examination, his vitals and blood pressure were stable. On examination, there was thinning of hair follicle with associated hair loss. Another system examination was normal. There were no other associated causes of hair loss in this patient. Hence, the clinician suspected warfarininduced hair loss and other drugs causing hair loss were ruled out. Hence, diagnosis of warfarin-induced alopecia was made and warfarin was not stopped.

\section{DISCUSSION}

Warfarin, an oral anticoagulant acts by inhibiting the clotting Factors II, VII, IX, and X. It acts by inhibiting the enzyme Vitamin K epoxide reductase thereby preventing the gamma carboxylation of clotting factors. Gamma carboxylation is required for clotting factors to get activated. Warfarin is, therefore, considered as Vitamin K antagonist. It is indicated for treatment and prevention of venous thrombosis and to prevent thrombotic complication due to atrial fibrillation. It is also used to treat patients following prosthetic heart valve replacement surgeries, recurrent myocardial infarction and also the treatment of transient ischemic attacks. The primary goal of warfarin treatment is to maintain the target international normalized ratio [3]. Complications of warfarin include nausea, diarrhea, vomiting, elevation of liver enzymes, renal nephropathy, skin necrosis, purple toe syndrome, bleeding complications, leukopenia, and agranulocytosis [4]. Only a few cases have been reported worldwide on warfarin-induced alopecia. As per literature search, it was found, 14 case reports of warfarin-induced alopecia published so far [5]. Hair fall due to the drug is an idiosyncratic adverse reaction of unknown mechanism. The first case of warfarin-induced alopecia was reported in a 2-year-old girl following ingestion of rat poison, which has warfarin as the active ingredient. Hair fall is also associated with other oral anticoagulants and as per WHO-Uppsala Monitoring Centre VigiBase877 cases of warfarin alopecia had been recorded [5].

Mechanism attributed to warfarin-induced hair fall includes antimitotic effect on epithelial follicular growth, stimulation of epidermal proliferation and inhibition of epithelial bulb cell proliferation. TE is strongly suspected as the mechanism due to timing and presentation of hair loss induced by warfarin. Another mechanism attributed to warfarin-induced hair loss includes change in vasculature of scalp leading to focal degeneration of the connective near the blood vessels of follicular connective tissue sheath $[3,5]$. It is also noted that when the hair follicles enter the telogen phase asynchronously following chronic exposure of drugs, the hair fall becomes clinically significant only after months or years of treatment with the offending drug. As per previous literature evidence, the alopecia caused by warfarin is usually reversible and it reappears on rechallenge. The time range of warfarininduced alopecia is from 3 months to 13 years [5]. There are more reports with women having alopecia following warfarin compared to men. This gender variation is attributed to frequent grooming of hair by females which might aggravate the shedding of the resting hair resulting from TE [5].

In our case, hair fall was noted in female patient following warfarin administration after almost 8 years and other causes were ruled out. Hence, a causality assessment was done using Naranjo et al.'s scale [6] and a probable causal relationship was ascribed.

\section{CONCLUSION}

Since many cases of alopecia due to oral anticoagulants have been reported and whether alopecia is dose related is still unclear, proper monitoring during anticoagulant therapy is of foremost concern. Further, prospective trials can be done to find out the exact incidence and prevalence of alopecia due to anticoagulant therapy in Southern India. 


\section{REFERENCES}

1. Gordon KA, Tosti A. Alopecia: Evaluation and treatment. Clin Cosmet Investig Dermatol 2011;4:101-6.

2. Lesiak K, Bartlett JR, Frieling GW. Drug-Induced Alopecia. In cutaneous drug eruptions. London: Springer; 2015. p. 215-27.

3. Teles JS, Fukuda EY, Feder D. Warfarin: Pharmacological profile and drug interactions with antidepressants. Einstein (Sao Paulo) 2012;10(1):110-5.
4. Pirmohamed M. Warfarin: Almost 60 years old and still causing problems. Br J Clin Pharmacol 2006;62(5):509-11.

5. Watras MM, Patel JP, Arya R. Traditional anticoagulants and hair loss: A role for direct oral anticoagulants? A review of the literature. Drugs Real World Outcomes 2016;3(1):1-6.

6. Naranjo CA, Busto U, Sellers EM, Sandor P, Ruiz I, Roberts EA, et al. A method for estimating the probability of adverse drug reactions. Clin Pharmacol Ther 1981;30(2):239-45. 